\title{
Exploitation of spatial information in high resolution digital imagery to map leaf area index
}

\author{
C. L. Walthall · Y. Pachepsky • W. P. Dulaney • D. J. Timlin • \\ C. S. T. Daughtry
}

Published online: 18 November 2007

(C) Springer Science+Business Media, LLC 2007

\begin{abstract}
Crop responses to management practices and the environment, as quantified by leaf area index (LAI), provide decision-making criteria for the delineation of crop management zones. The objective of this work was to investigate whether spatial correlations inferred from remotely sensed imagery can be used to interpolate and map LAI using a relatively small number of ground-based LAI measurements. Airborne imagery was recorded with the Airborne Imaging Spectrometer for Applications (AISA) radiometer over a 3.2 ha corn field. Spectral vegetation indexes (SVI) were derived from the image and aggregated to cells of $2 \times 2 \mathrm{~m}^{2}, 4 \times 4 \mathrm{~m}^{2}$, and $8 \times 8 \mathrm{~m}^{2}$ resolution. The residual maximum likelihood method was used to estimate the LAI variogram parameters. A generalized least squares regression was used to relate ground truth LAI data and collocated image pixels. This regression result was then used to convert variograms from the imagery to LAI units as well as to interpolate and map LAI. The decrease in resolution by merging pixels led to an increase in the value of the $r^{2}$ and to a decrease in root meansquared error (RMSE) values. The accuracy of kriged estimates from the variogram of the measured LAI and that from the image derived variograms was estimated by cross-validation. There was no difference in the accuracy of the estimates using either variograms from measured LAI values or from those of converted SVIs. Maps of LAI from groundbased measurements made by kriging the data with image-derived variogram parameters were similar to those obtained by with kriging with the variogram of measured LAI. Similar coarse spatial trends of high, medium and low LAI were evident for both maps.
\end{abstract}

C. L. Walthall

USDA-ARS, Beltsville, USA

W. P. Dulaney · C. S. T. Daughtry

Hydrology and Remote Sensing Laboratory, USDA-ARS, Beltsville, USA

Y. Pachepsky ( $ه)$

Environmental Microbial Safety Laboratory, USDA-ARS, Bldg. 173 Powder Mill Road, BARC-EAST, Beltsville, MD 20705, USA

e-mail: Yakov.Pachepsky@ars.usda.gov

D. J. Timlin

Crop Systems and Global Change Laboratory, USDA-ARS, Beltsville, USA 
Variogram parameters from ground-based measurements of LAI compared favorably with those derived from remotely sensed imagery and could be used to provide reasonable results for the interpolation of LAI measurements.

Keywords Leaf area index - Spectral vegetation index · Variogram .

Mapping · Cross-validation · Residual maximum likelihood method (REML)

\section{Introduction}

Maps that describe accurately the spatial distribution of important agronomic properties are essential for a wide variety of applications, such as the delineation of management zones for precision farming. Foliage density, expressed as leaf area index (LAI), is a useful property for quantifying the spatial variation of factors that affect crop productivity. As a standardized biophysical quantity, LAI can be compared from one location to another, and within and between growing seasons.

The number of ground measurements required to map LAI accurately by traditional ground-based sampling approaches can be prohibitively expensive. Kerry and Oliver (2003) discussed a similar situation with respect to soil mapping for precision agriculture and noted that the precision agriculture literature has long suggested using ancillary information that is more intensive and cheaper to obtain (Blackmer et al. 1995; Mulla 1997; Leon et al. 2003).

High spatial resolution digital imagery capable of recording within-field agronomic variation is becoming more available commercially, and is thus becoming an attractive alternative for mapping LAI. Techniques for mapping LAI from remotely sensed data include: (1) calibration of imagery with field measurements, (2) radiative transfer model inversion and (3) hybrid methods that combine radiative transfer modeling with limited calibrated field measurements (Walthall et al. 2004b; Fang et al. 2003).

The most common approach to mapping LAI uses an ordinary least squares (OLS) regression equation between ground-based destructive or non-destructive LAI calibration measurements and a remotely sensed spectral vegetation index (SVI), such as the Normalized Difference Vegetation Index (NDVI). These data are used to transform an SVI image into a quantified LAI image map (e.g., Johnson et al. 2001). The OLS 'SVI vs. LAI' regression is essentially a point-based relationship and applying it to each image pixel does not ensure that an accurate map of LAI distribution will result. To overcome this limitation, ancillary data and spatial data of interest should be spatially correlated if the former is to be used to improve the resulting map of the latter. Similar problems have been reported previously in the agronomic literature. For example, OLS regression showed that soil hydraulic properties were reasonably well related to common soil properties, yet the spatial structures of estimated and measured hydraulic parameters appeared to be quite different (Sinowski et al. 1997; Romano 2004).

Kerry and Oliver (2003) have shown that variograms of ancillary data can be used to aid sampling for soil surveys when the ranges of spatial dependence of both soil properties and remote sensing data are of similar orders of magnitude and when maps show similar patterns of spatial variation in the ancillary and soil data. The objective of this study was to investigate whether spatial correlations inferred from remotely sensed imagery can be used with a relatively small number of ground-based LAI samples to produce reasonably accurate maps of LAI. 


\section{Materials and methods}

The study site is a 22 ha corn field at the USDA, Beltsville Agricultural Research Center in Beltsville, Maryland ( $39^{\circ} 01^{\prime} 00^{\prime \prime} \mathrm{N}, 76^{\circ} 52^{\prime} 00^{\prime \prime} \mathrm{W}$; Fig. 1). A digital elevation map (DEM) developed using kinematic global positioning system (GPS) data acquired on a 5-m grid showed that $74 \%$ of the site has a slope $<2 \%$ and only $2 \%$ of the site has a slope $>3 \%$. The soil has a sandy texture with buried clay lenses. The representative soil taxonomy is the coarse-loamy, siliceous, mesic, Typic (or Aquic) Hapludult.

Corn (Zea mays L. 'Pioneer 33Y18') was planted on May 29, 1999 using the best management practices for rain-fed corn in Maryland which included $30 \mathrm{~kg} \mathrm{~N} \mathrm{ha}^{-1}$ applied at planting and $130 \mathrm{~kg} \mathrm{~N} \mathrm{ha}^{-1}$ applied as a side dressing on July 1st. The spacing of the rows was $0.76 \mathrm{~m}$. Weeds were controlled with pre-emergence herbicides. The groundbased LAI measurements were acquired within 48 hours of the airborne data collection using non-destructive optical methods with an LAI-2000 Plant Canopy Analyzer (PCA; Licor, Inc., Lincoln, NE). The sample sites were selected randomly from a reference grid established using differential GPS (DGPS) and marked with annotated flags after planting. At each site, two series of 'one above canopy' and 'four below canopy' readings were acquired at evenly spaced intervals along diagonal transects between adjacent rows either early in the morning or late afternoon when the sun was near the horizon or obscured by clouds. Heights of five plants were measured on 21 July 1999 near each flag, Mean plant height ranged from 1.6 to $2.4 \mathrm{~m}$. The sample area radius of the PCA is reported to be about three times the height of the canopy (LiCor 1992), therefore, the sample support was about $6 \mathrm{~m}$ for the data used here. The LAI data were checked manually for outliers and their values were consistent with those in corresponding locations measured in previous years. The ground-based LAI data were kriged by ordinary kriging to the same resolution as the image data to enable comparison with the image-derived LAI results. The variogram model parameters of LAI were used with the data to obtain the weights that are an essential component of the optimal estimation procedure.

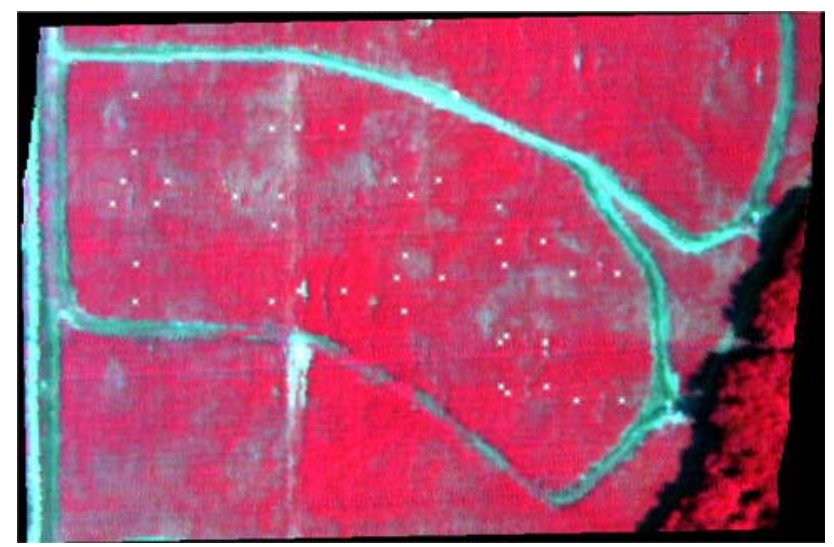

Fig. 1 False color image of the study site at the Beltsville Agricultural Research Center, Beltsville, MD taken with the AISA airborne scanner. The field is sub-watershed B of the Optimizing Production inputs for Economic and Environmental Enhancement (OPE3) study site and covers approximately 3.4 ha. The white dots denote locations of the ground-based non-destructive LAI measurements. The effects of soil compaction on crop production from an abandoned road that ran $\mathrm{N}-\mathrm{S}$ through the site are visible as the lighter-toned vertical feature just to the left of image center 
Non-destructive, optically-based LAI procedures use relationships between foliage density and canopy light transmittance and do not differentiate between brown and green LAI, or between leaves and non-leaf canopy constituents such as petioles and stems (Welles and Norman 1991). The algorithms used in the above procedures also assume azimuthally randomly oriented foliage that are free from clumping effects which have been shown to contribute potentially significant error terms (Lacaze et al. 2002; Lacaze and Roujean 2001). Canopy reflectance pixels contain a mix of reflectance contributions from canopy and non-canopy elements including underlying soil and crop residue materials (Norman et al. 1985). Thus, both types of data contain errors that might limit the correlation of any SVI derived from canopy reflectance with foliage density derived from canopy transmittance data.

Airborne imagery was recorded with the Airborne Imaging Spectrometer for Applications (AISA) on July 16th, 1999 (Braam et al. 1993) from approximately $330 \mathrm{~m}$ above ground level (AGL) under clear skies. This provided a ground instantaneous field of view (IFOV) of $1 \mathrm{~m}$. Raw pixel values for the $10 \mathrm{~nm}$ wide spectral bands centered at 672 and $808 \mathrm{~nm}$ were calibrated to absolute at-sensor radiance using coefficients derived from laboratory based measurements. Upward-looking sensor values were taken simultaneously with downward-looking measurements via a diffuser attached to a fiber optics cable mounted through the top of the aircraft cabin. Apparent reflectance factors were calculated by dividing the downward-looking pixel values by the upward-looking sensor readings. Apparent reflectance was used to calculate NDVI for each pixel

$$
\mathrm{NDVI}=[(\mathrm{NIR}-\mathrm{Red}) /(\mathrm{NIR}+\mathrm{Red})] .
$$

where NIR is near infrared.

First-order geometric corrections based on GPS and inertial navigation unit data were done on the imagery after its acquisition. Minor georegistration errors were corrected by 'rubbersheeting' of the imagery to very accurate ground control points. The remotely sensed data were aggregated from the original $1 \times 1 \mathrm{~m}^{2}$ pixels to $2 \times 2 \mathrm{~m}^{2}, 4 \times 4 \mathrm{~m}^{2}$ and $8 \times 8 \mathrm{~m}^{2}$ pixels by averaging.

The LAI maps for 2, 4 and 8-m pixel sizes produced by linear regression of the SVI values on the collocated ground-based LAI data were examined to determine whether OLS or Generalized Least Squares (GLS) 'SVI versus LAI' regression was the more appropriate. The OLS regression assumes that the errors are random and independent and it does not take account of spatially correlated regression errors even though they are likely when biophysical parameters are sampled at close distances. Spatially correlated regression errors are known to result in biased regression coefficients and predictions (Pinheiro and Bates 2000). The use of GLS offers an attractive alternative to OLS because it accounts for spatially correlated regression errors. (Spatially correlated errors were present in a $1 \times 1 \mathrm{~m}^{2}$ pixel data set used by Walthall et al. (2001)). The SPLUS software package (Mathsoft 1999) was used to compute the GLS regressions. The GLS regression equations were then used to convert the SVI values to LAI values.

All geostatistical computations were done using the package geoR in the $R$ software system (Ribeira and Diggle 2001). We used the residual maximum likelihood method (REML) as coded in the geoR subroutine 'likfit' to estimate the variogram and model parameters from the original and converted SVI data of LAI. There are few ground LAI data compared with the SVI data and Kerry and Oliver (2005) showed that estimation of the variogram by REML is efficient for relatively small sets of data. This method also provides unbiased estimates of variances and covariances because it uses the error 
contrasts which are free from any fixed effects in the model. The variogram parameters of the converted SVI data were used to interpolate the ground truth data by kriging. The accuracy of such estimates was evaluated by cross-validation and compared with the accuracy of those based on the variogram of the ground LAI data.

\section{Results}

Figure 2 a shows the variogram estimated by REML. An exponential function satisfied the criterion of minimizing the negative log-likelihood function. The nugget value approached zero in all 10 of the implementations of the program with different initial nugget, sill and range estimates. The best model based on the above criterion had the distance parameter of $17.47 \mathrm{~m}$ (an approximate range of $52.4 \mathrm{~m}$ ) and a sill of 0.235 (Table 1).

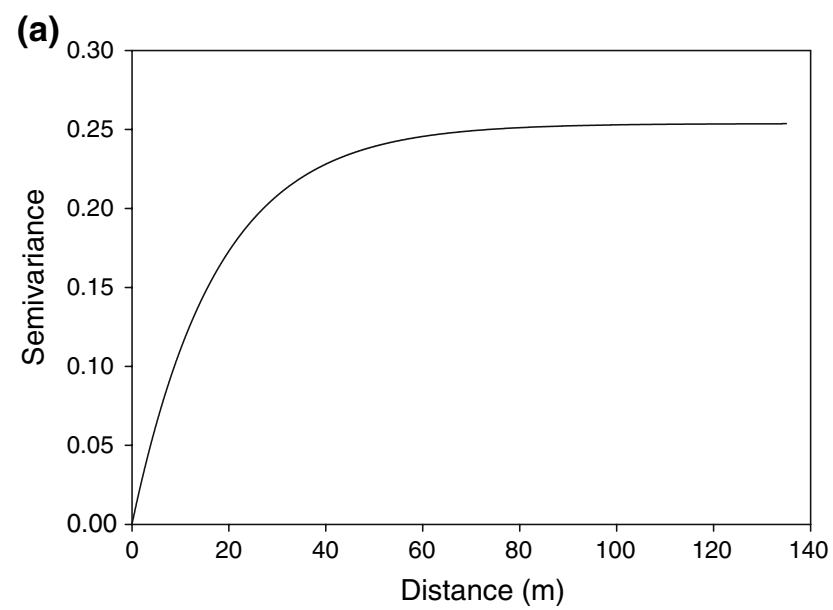

(b)

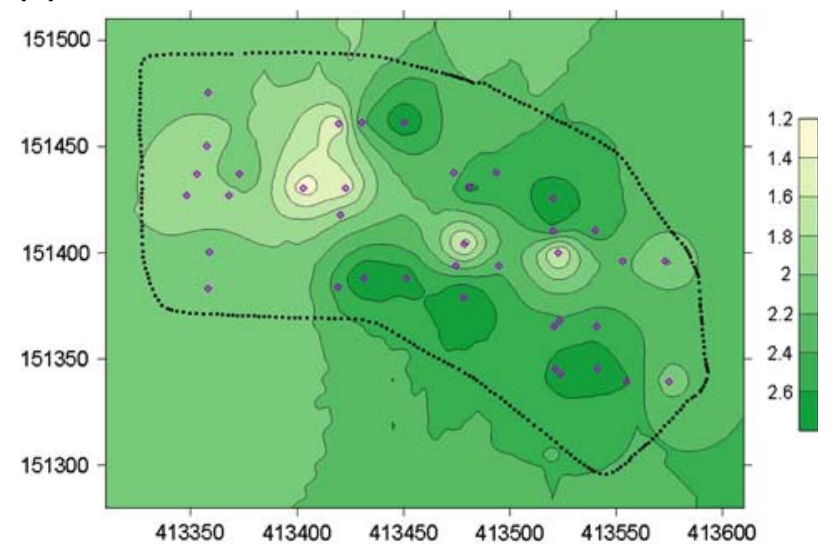

Fig. 2 Leaf area index (LAI) from the ground measurements: (a) variogram of LAI estimated by REML and fitted by an exponential function, and (b) map of LAI produced by ordinary kriging of the ground measurements with the REML variogram 
Table 1 Parameters of the exponential variograms ${ }^{\mathrm{a}}$ and the root-mean-squared error (RMSE) of the interpolation of LAI by kriging

\begin{tabular}{llll}
\hline Data & Distance parameter (approximate variogram range $\left.{ }^{\mathrm{c}}\right), \mathrm{m}$ & Sill variance & RMSE $^{\mathrm{b}}$ \\
\hline Measured LAI & $17.5(52.5)$ & 0.254 & 0.430 \\
$\mathrm{SVI}, 2 \times 2 \mathrm{~m}^{2}$ & $16.9(50.7)$ & 0.00462 & 0.430 \\
$\mathrm{SVI}, 4 \times 4 \mathrm{~m}^{2}$ & $17.0(51.0)$ & 0.00432 & 0.430 \\
$\mathrm{SVI}, 8 \times 8 \mathrm{~m}^{2}$ & $17.0(51.0)$ & 0.00401 & 0.427 \\
\hline
\end{tabular}

\footnotetext{
a Nugget variance is zero for all variograms

b From cross-validation

c The approximate variogram range (in parentheses) is equal to three times the distance parameter for the exponential variogram
}

Figure $2 b$ shows the map of ordinary kriged estimates of LAI made from the ground measurements and the variogram parameters given in Table 1. The range of LAI values is from 1.2 to 2.6 with isolated areas of small and large values. The largest LAI values occur in the eastern part of the field and small and moderate values dominate the western part. A trough of mid-range LAI values extends from $\mathrm{W}$ to $\mathrm{E}$ through the middle of the larger LAI values.

Figure 3 shows the REML variograms of SVI computed from the image data for the range of pixel sizes $(2,4$ and $8-\mathrm{m})$. As above the exponential function provided the best fit with zero nugget variance. The sill variances decrease as the pixel sizes increase as one would expect as an increase in the sample support generally reduces the variance. A doubling of the pixel size resulted in a decrease of about $7 \%$ of the sill value of the $1-\mathrm{m}$ pixel data (Table 1). The model parameters are given in Table 1. The distance parameters of the models for all pixel sizes are about $17.0 \mathrm{~m}$ and close to that of the ground LAI variogram.

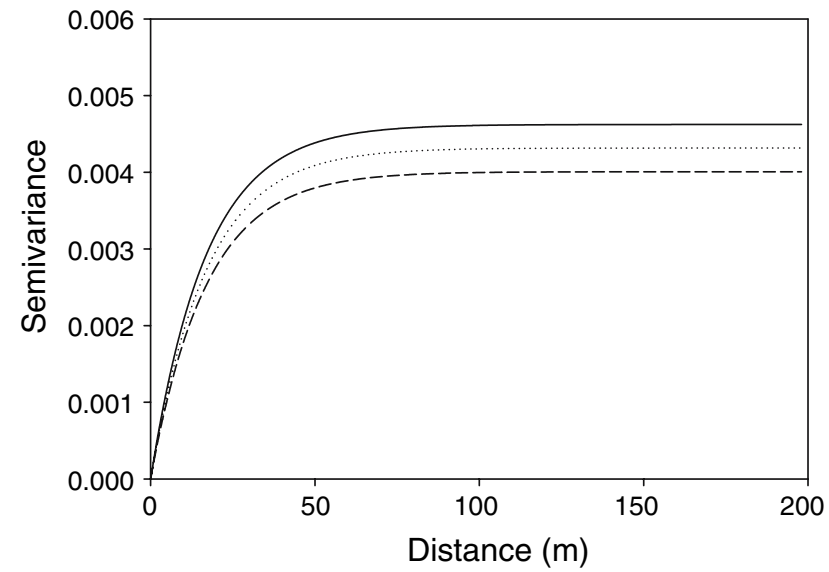

Fig. 3 Variograms of the SVI imagery estimated by REML for pixel sizes of 2-m ( ), 4-m (.....), and 8-m (_ _ _ _ $)$ 
Figure 4 shows the relationships between collocated LAI and SVI values. The linear regression equations were:

$$
\mathrm{LAI}=4.751 \times \mathrm{SVI}-1.323 ; r^{2}=0.495 ; \mathrm{RMSE}=0.340
$$

for the 2-m pixel size,

$$
\mathrm{LAI}=5.536 \times \mathrm{SVI}-1.911 ; r^{2}=0.557 ; \mathrm{RMSE}=0.322
$$

for the 4-m pixel size and

$$
\mathrm{LAI}=6.688 \times \mathrm{SVI}-2.766 ; r^{2}=0.657 ; \mathrm{RMSE}=0.286
$$

for the $8-\mathrm{m}$ pixel size. Here $r^{2}$ is the coefficient of determination of the regression and RMSE is the root mean-squared error. One datum was removed from the 2-m data because it was judged to be an outlier. (The value of $r^{2}$ was 0.331 before this outlier was removed). The increase in the pixel size led to an increase in the $r^{2}$ values and to a moderate decrease in RMSE values. The accuracy of the SVI vs. LAI regression increased slightly as the pixel size increased (Fig. 4).

The accuracy of kriged estimates for the ground-based LAI was assessed by crossvalidation using the REML variogram of those data (Fig. 2a, Table 1). The mean error was -0.006 and the mean RMSE was 0.430 . Cross-validation was also used to evaluate the accuracy of using the image-derived variograms for spatial interpolation of important crop parameters, in this case LAI. The SVI values were converted to the LAI values by regression Eqs. 1-3 at all the measurement sites. Variograms of LAI derived from SVI were estimated by REML and are shown in Fig. 5. The sills of these variograms appear to depend on the pixel size, but they increase as the pixel size increases: 0.105 (2-m pixel), 0.134 (4-m pixel) and 0.181 (8-m pixel) (Fig. 5). This happens because of the substantial increase in the slopes of regressions (1)-(3) with the increase in pixel size. Sill values of the derived LAI variograms are less than that of the ground-measured LAI values (Table 1). Distance parameters of the derived LAI variograms are similar for the range of pixel sizes (Table 1).

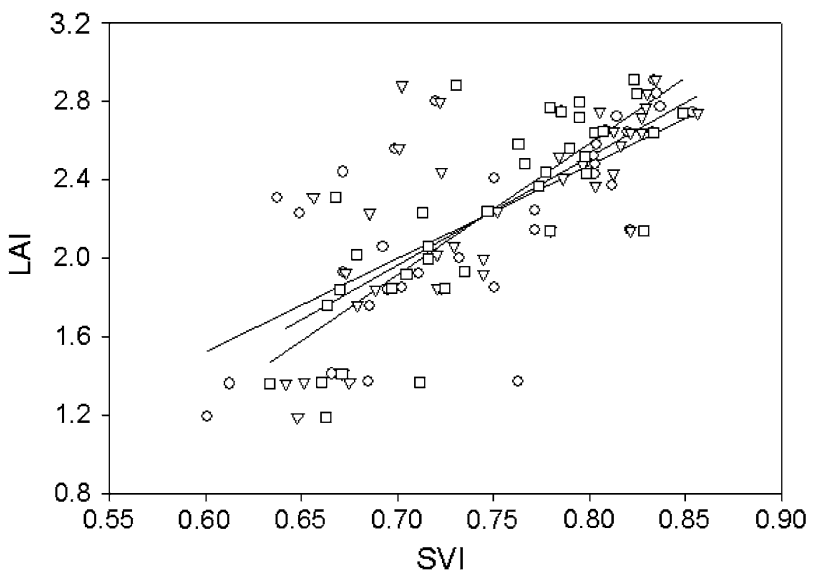

Fig. 4 Relationships between SVI estimated for different pixel sizes and measured LAI; o, $\nabla$, and $\square$ for pixel sizes of 2, 4, and 8-m, respectively 


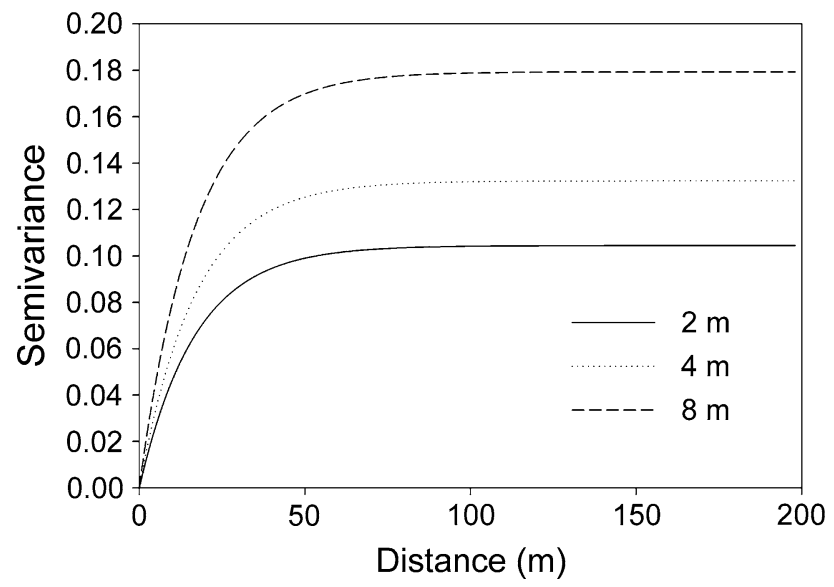

Fig. 5 Variograms of LAI derived from SVI estimated by REML for pixel sizes as listed in the legend and fitted by exponential functions

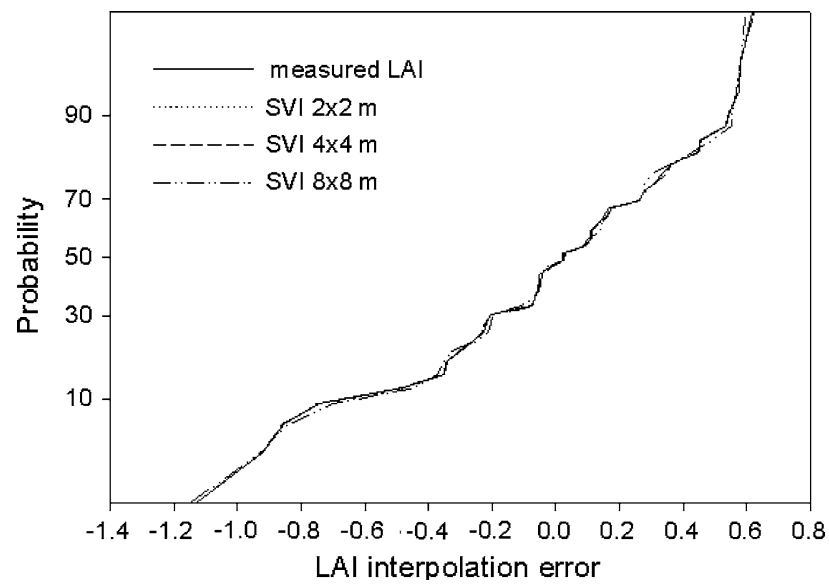

Fig. 6 Probability distributions of LAI interpolation errors obtained from cross-validation with different variograms. Sources of data for the variograms are shown in the legend

Differences in the sill values of derived LAI variograms for different pixel sizes when used to estimate LAI by kriging at each measurement point appear to have had little or no effect on the accuracy of the estimates. Table 1 gives the RMSEs for these variograms and data. Probability distributions of LAI estimation errors obtained by cross-validation are similar for all variograms, i.e. the variogram of LAI and variograms obtained by converting SVI to LAI for all pixel sizes (Fig. 6).

\section{Discussion}

Although the number of collocated values between the SVI pixels and ground-based LAIs was small, the SVI variograms could be used to estimate LAI. Kerry and Oliver (2003) 
observed a similar effect when they used dense soil $\mathrm{EC}_{\mathrm{a}}$ data and aerial photograph information as ancillary data to design an effective soil sampling scheme using geostatistics. It is possible that some aspects of the variation of both SVI and LAI have a similar spatial structure which results in their variograms being similar. Kerry and Oliver (2003) suggest that if densely-measured ancillary variables are available, it is sometimes possible to improve the accuracy of geostatistical estimation.

The RMSEs of regressing SVI versus LAI showed an increase in accuracy as the pixel size increased. The effect of pixel size on the strength of the relation between measured vegetation properties and remotely sensed imagery was also observed for grasslands by $\mathrm{He}$ et al. (2006). On the other hand, Colombo et al. (2003) found no change in accuracy for pixel resolutions of 12,24 and $36 \mathrm{~m}$. The spatial resolution of the image is expected to have an impact on the quality of the biophysical properties retrieved from remotely sensed imagery (Walthall et al. 2001; $\mathrm{Hu}$ and Islam 1997). A basic assumption of radiative transfer theory that underpins the retrieval of properties from most remote sensing procedures is that individual canopy and background elements that comprise a pixel are infinitely small compared to the dimension of the pixel itself (Walthall et al. 2004a; Goel 1988). One can usually assume that a crop image pixel comprises sunlit and shaded foliage as well as sunlit and shaded soil or plant residue. Therefore, it is possible that very high spatial resolution pixels are more likely to violate this basic assumption. Pixel values that are primarily related to the soil component will add significant bias and noise to the desired vegetation signal.

The pixel size did not affect the distance parameter (and the approximate range) of the image variograms in this study (Table 1). Although an increase in the variogram range with increasing pixel size or sample support is expected (Dungan 2001), relatively small changes in the support did not increase the range in other geostatistical studies (Dungan 2001; Fassnacht and Deems 2005; Skoien and Blöschl 2006).

The SVI image data did not capture all of the spatial variation in the factors affecting LAI in the field in this study, because the ground measured LAI had a larger variogram sill compared with the sills of derived LAI variograms. Moreover, a smoothing effect can be expected if the derived variograms are used to interpolate LAI from the 'ground truth' data. Based on these observations, a correction of the sill values from the imagery based on the variance of the LAI values across the field might be of value.

The use of image-derived variograms to krige ground-based measurements provided RMSEs that were commensurate with the kriged 'ground truth' LAI estimates. The significance of the LAI estimation errors of about 0.4 for LAI values ranging from 0 to 3 depends on the specific application. This degree of error might be acceptable if the goal is to delineate within-field areas with a consistent response to soil or other environmental factors. For applications, such as the input of LAI values to models to calculate the energy balance components or crop yield, etc., this level of error might not be tolerable.

Given the continuous spatial cover in remotely sensed imagery, it is possible that the image-derived spatial patterns might be more representative of the variation in LAI than the measured values. This raises the recurring question of how many ground samples are needed to provide an adequate representation of the spatial variation in LAI, to calibrate it, and to provide robust validation of alternative methods for mapping LAI. Progress in this area hinges on developing metrics for the quantitative comparison of spatial patterns. Such developments would be welcomed given precision farming's reliance at present on management strategies that use zones within fields for the analysis of soil and crop variability as well as the guidance of agricultural machinery for variable-rate applications. 


\section{Conclusion}

The use of variograms derived from remotely sensed imagery to represent LAI provided reasonable results when used to interpolate measured LAI values in this study. Kriged maps of ground-based LAI measurements made using model parameters of image derived variograms were comparable to those obtained by kriging with variograms computed from the measured LAI values. Similar spatial patterns of high, medium and low LAI for both sets of maps were evident. The capability of image-derived variograms to approximate that based on measured LAI is limited by the strength of the correlation between LAI and SVI. An examination of the point accuracy of LAI maps as measured by the RMSE from crossvalidation confirmed that the results improved with decreasing spatial resolution for pixel dimensions up to $8 \mathrm{~m}$ by $8 \mathrm{~m}$ in this study. The results described in this paper, albeit limited to the single site and the single ancillary variable, show that the use of remotely sensed imagery to estimate spatial patterns of plant leaf area index is a promising avenue for further research.

Acknowledgements The authors gratefully acknowledge the assistance of Andrew Russ during the collection and processing of the LAI-2000 PCA data, and Bill Bernard, Oliver Weatherbee and Lee Watson for the calibration, collection and processing of the AISA imagery. The invaluable help of Margaret Oliver and advice from anonymous reviewers is highly appreciated.

Disclaimer Mention of company names is for information purposes only and does not constitute endorsement to the exclusion of others.

\section{References}

Blackmer, T. M., Schepers, J. S., \& Meyer, G. (1995). Remote sensing to detect nitrogen deficiency in corn. In P. C. Robert, R. H. Rust, \& W. E. Larson (Eds.), Proceedings of site-specific management for agricultural systems. Second international conference (pp. 505-512). Madison: ASA-CSSA-SSSA.

Braam, B., Okkonen, J., Aikio, M., Makisara, J., \& Bolton, J. (1993). Design and first test results of the Finnish airborne image spectrometer for different applications. In G. Vane (Ed.), Proc. of spectrometry of the terrestrial environ (pp. 142-151), (SPIE 197).

Colombo, R., Bellingeri, D., Fasolini, D., \& Marino, C. M. (2003). Retrieval of leaf area index in different vegetation types using high resolution satellite data. Remote Sensing of Environment, 86, 120-131.

Dungan, J. L. (2001). Scaling up and scaling down: The relevance of the support effect on remote sensing of vegetation. In: N. J. Tate, \& P. M. Atkinson (Eds.), Modeling scale in geographical information science (pp. 221-236). Hoboken: Wiley and Sons.

Fang, H., Liang, S., Chen, M., Walthall, C. L., Daughtry, C. S. (2003). Statistical comparison of MISR, ETM+ and MODIS land surface reflectances and albedo productions of the BARC land validation core site, USA. International Journal of Remote Sensing, 25, 409-422.

Fassnacht, S. R., \& Deems, J. S. (2005). Scaling associated with averaging and resampling of LiDARderived Montana snow depth data. In: Proceedings of the 62nd Eastern Snow Conference, pp. 163-172.

Goel, N. S. (1988). Models of vegetation canopy reflectance and their use in estimation of biophysical parameters from reflectance data. Remote Sensing Reviews, 4, 1-222.

He, Y., Guo, X., Wilmshurst, J., \& Si, B. C. (2006). Studying mixed grassland ecosystems II: optimum pixel size. Canadian Journal of Remote Sensing, 32, 108-115.

Hu, Z., \& Islam, S. (1997). A framework for analyzing and designing scale invariant remote sensing algorithms. IEEE Transactions on Geoscience and Remote Sensing, 35, 747-755.

Johnson, L. F., Roczen, D., \& Youkhana, S. (2001). Vineyard canopy density mapping with Ikonos satellite imagery. In: Proc. Third International Conference on Geospatial Information in Agriculture and Forestry, Denver, Colorado, 5-7 November 2001, 10 pp.

Kerry, R., \& Oliver M. A. (2003). Variograms of ancillary data to aid sampling for soil surveys. Precision Agriculture, 4, 261-278. 
Kerry, R., \& Oliver, M. A. (2005). Maximum likelihood variograms for efficient prediction in precision agriculture. In J. V. Stafford (Ed.), Precision agriculture '05 (pp. 707-714). The Netherlands: Wageningen Academic Publishers.

Lacaze, R., Chen, J. M., Roujean, J.-L., \& Leblanc, S. G. (2002). Retrieval of vegetation clumping index using hot spot signatures measured by POLDER instrument. Remote Sensing of Environment, 79, 84-95.

Lacaze, R., \& Roujean, J.-L. (2001). G-function and Hot SpoT (GHOST) reflectance model application to multi-scale airborne POLDER measurements. Remote Sensing of Environment, 76, 67-80.

Leon, C., Shaw, D. R., Cox, M. S., Abshire, M. J., Ward, B., Wardlaw, M. C., \& Watson, C. (2003). Utility of remote sensing in predicting crop and soil characteristics. Precision Agriculture, 4, 359-384.

LiCor. (1992). LAI-2000 plant canopy analyzer operating manual. Lincoln: Li-Cor.

Mulla, D. J. (1997). Key processes and properties for site specific soil and crop management. In: F. J. Pierce, \& E. J. Sadler (Eds.), The state of site specific management for agriculture (pp. 1-18). Madison: ASA-CSSA-SSSA.

Norman, J. M., Welles J. M., \& Walter-Shea, E. A. (1985). Contrasts among bidirectional reflectance of leaves, canopies, and soils. IEEE Transactions on Geoscience and Remote Sensing, 23, 659-667.

Pinheiro, J. C., \& Bates, D. M. (2000). Mixed-effects models in S and S-Plus. New York: Springer.

Ribeiro. J. R., \& Diggle, P. J. (2001). geoR: A package for geostatistical analysis. R-NEWS Vol 1, No 2. ISSN 1609-3631.

Romano, N. (2004). Spatial structure of PTF estimates. In Y. A. Pachepsky, \& W. J. Rawls (Eds.), Development of pedotransfer functions in soil hydrology (pp. 295-322). Amsterdam, The Netherlands: Elsevier.

Sinowski, W., Scheinost, A. C., \& Auerswald, K. (1997). Regionalization of soil water retention curves in a highly variable soilscape, II. Comparison of regionalization procedures using a pedotransfer function. Geoderma, 78, 145-149.

Skoien, J. O., \& Blöschl, G. (2006). Scale effects in estimating the variogram and implications for soil hydrology. Vadose Zone Journal, 5, 153-167.

Walthall, C., Dulaney, W., Anderson, M., Norman, J., Fang, H., \& Liang, S. (2004a). A comparison of empirical and neural network approaches for estimating corn and soybean leaf area index from Landsat ETM+ imagery. Remote Sensing of Environment, 92, 465-474.

Walthall, C. L., Dulaney, W. P., Anderson, M., Norman, J., Fang, H., Liang, S., Timlin, D., \& Pachepsky, L. (2004b). Alternative approaches for estimating leaf area index (LAI) from remotely sensed satellite and aircraft imagery. In: Proceedings of Society of Photonics Instrumentation Engineers. International Symposium on Optical Science and Technology 2-6 August, 2004,Denver, CO.

Walthall, C. L., Kaul, M., Timlin, D. J., \& Daughtry, C. S. T. (2001). Linking within-field response with soil characteristics to define crop response zones for management zone delineation. In: Proc. Third International Conference on Geospatial Information in Agriculture and Forestry, Denver, CO, CD-ROM.

Welles, J. M., \& Norman, J. M. (1991). Instrument for indirect measurement of canopy architecture. Agronomy Journal, 83, 818-825. 